\title{
Combining Methods of Lyapunov for Exponential Stability of Linear Dynamic Systems on Time Scales
}

\author{
Nguyen Ngoc Huy1, Dang Dinh Chau² \\ ${ }^{1}$ Department of Mathematics, Vietnam Water Resource University, Hanoi, Vietnam \\ ${ }^{2}$ Department of Mathematics, Vietnam National University of Science, Hanoi, Vietnam \\ Email: huynn@wru.edu.vn, chaudida@gmail.com
}

Received 27 September 2014; revised 20 October 2014; accepted 2 November 2014

Copyright (C) 2014 by authors and Scientific Research Publishing Inc.

This work is licensed under the Creative Commons Attribution International License (CC BY).

http://creativecommons.org/licenses/by/4.0/

c) (i) Open Access

\section{Abstract}

Consider the linear dynamic equation on time scales

$$
x^{\Delta}(t)=A(t) x(t)+f(t, x) ; x\left(t_{0}\right)=x_{0}, t \in\left[t_{0}, \infty\right)_{T},
$$

where $x(.) \in R^{n}, A(.) \in C_{r d}\left(\left[t_{0}, \infty\right)_{T}, M_{n}(R)\right), f:\left[t_{0}, \infty\right)_{T} \times R^{n} \rightarrow R^{n}$ is a rd-continuous function, $T$ is a time scales. In this paper, we shall investigate some results for the exponential stability of the dynamic Equation (1) by combinating the first approximate method and the second method of Lyapunov.

\section{Keywords}

Time Scales, Exponential Stability, Linear Dynamic Equation

\section{Introduction}

Let $R^{n}$ be a $n$-dimension Euclidean space, $T$ be a time scales (a nonempty closed subset of $R$ ). We denote $T_{\alpha}^{+}:=[\alpha, \infty) \cap T$. For convenience, we shall use the notions which appear in the book by Bohner and Peterson (see [1] [2]). The notions related to the Lyapunov function that we use follow the results of B. Kaymakcalan (see [3]). For necessary, we recall them in this process.

We consider a dynamic equation

$$
x^{\Delta}(t)=F(t, x), t \in T_{t_{0}}^{+},
$$


where $F \in C_{r d}\left(T_{t_{0}}^{+}, R^{n}\right)$ with $F(t, 0)=0$. We suppose that $F$ satisfies all conditions such that (2) has a unique solution $x\left(t, t_{0}, x_{0}\right)$ with $x\left(t_{0}\right)=x_{0}$. In this paper, we define the stable notions of the trivial solution $x(t)=0$ of (2) as the followings:

Definition 1. The trivial solution $x(t)=0$ of (2) is stable on $T_{t_{0}}^{+}$forall $\varepsilon>0$, there exists $\delta=\delta\left(\varepsilon, t_{0}\right)>0$ that satisfies $\left\|x_{0}\right\|<\delta$ then $\left\|x\left(t, t_{0}, x_{0}\right)\right\|<\varepsilon, \forall t \geq t_{0}$.

Definition 2. The trivial solution $x(t)=0$ of (2) is asymptotically stable if it is stable and there exists $\delta_{0}=\delta_{0}\left(t_{0}\right)>0$ satisfies $\left\|x_{0}\right\|<\delta_{0}$ then

$$
\lim _{t \rightarrow \infty}\left\|x\left(t, t_{0}, x_{0}\right)\right\|=0 \text {. }
$$

In these definitions, if the numbers $\delta$ and $\delta_{0}$ do not depend on $t_{0}$, we say that the trivial solution of (2) is uniformly stable (uniformly asymptotically stable).

Definition 3. The trivial solution $x(t)=0$ of (2) is exponential stable on $T_{t_{0}}^{+}$if there exists $K=K\left(t_{0}\right)>0$ and $q>0$ with $-q$ is positively regressive which satisfies

$$
\|x(t)\| \leq K e_{-q}\left(t, t_{0}\right)\left\|x_{0}\right\|, \quad \forall t \geq t_{0} .
$$

In the simple case (see [2]), consider the dynamic equation

$$
\left\{\begin{array}{l}
x^{\Delta}(t)=p(t) x(t) \quad t \in T_{t_{0}}^{+} \\
x\left(t_{0}\right)=x_{0}
\end{array}\right.
$$

The solution of (3) is exponential function $e_{p}\left(t, t_{0}\right)=\exp \left\{\int_{t_{0}}^{t} \lim _{s \rightarrow \mu(\tau)} \frac{\ln |1+p(\tau) s|}{s} \Delta \tau\right\}$. We recall some properties of the exponential function which are used later.

Assume $p, q \in C_{r d}\left(T_{t_{0}}^{+}, R\right)$, we denote

$$
\begin{gathered}
p \oplus q=p(t)+q(t)+\mu(t) p(t) q(t) \\
\Theta p=-\frac{p(t)}{1+\mu(t) p(t)} .
\end{gathered}
$$

We have the following equalities

1) $e_{0}(t, s)=1 ; e_{p}(t, t)=1 \forall t, s \in T$;

2) $e_{p}(\sigma(t), s)=(1+\mu(t) p(t)) e_{p}(t, s) \quad \forall t, s \in T$;

3) $e_{p}(t, s) e_{p}(s, \tau)=e_{p}(t, \tau), t \geq s \geq \tau \quad \forall t, s \in T$;

4) $\frac{1}{e_{p}(t, s)}=e_{\Theta p}(t, s) \forall t, s \in T$;

5) $e_{p}(t, s)=\frac{1}{e_{p}(s, t)}=e_{\Theta p}(s, t) \quad \forall t, s \in T$;

6) $e_{p}(t, s) e_{q}(t, s)=e_{p \oplus q}(t, s) \quad \forall t, s \in T$;

7) $\frac{e_{p}(t, s)}{e_{q}(t, s)}=e_{p \oplus q}(t, s) \quad \forall t, s \in T$.

In the special case $p(t)=\lambda(\lambda \in C)$, we have

$$
e_{\lambda}(t, s)=\exp \left\{\int_{s}^{t} \lim _{s \rightarrow \mu(\tau)} \frac{\ln |1+\lambda s|}{s} \Delta \tau\right\} .
$$

Using the notations

$$
S(T)=S_{R}(T) \cup S_{C}(T),
$$

where 


$$
\begin{gathered}
S_{C}(T)=\left\{\lambda \in C: \limsup _{\tau \rightarrow \infty} \frac{1}{\tau-t_{0}} \int_{t_{0}}^{\tau} \lim _{s \rightarrow \mu(t)} \frac{\ln |1+\lambda s|}{s} \Delta t<0\right\}, \\
S_{R}(T)=\{\lambda \in R: \forall \tau \in T, t>\tau, 1+\mu(t) \lambda=0\},
\end{gathered}
$$

$S(T)$ is set of exponential stability of $e_{\lambda}(t, s)$ (see [4]).

Theory of stability of dynamic equation on time scales is an area of mathematics that has recently received a lot of attention (see [1] [2] [4]-[7]). And almost of the results which involve the methods of Lyapunov to investigate the stability, have been developed and obtained the interesting results to expand for dynamic equation on time scales. Besides that the criterions and sufficient conditions were given, there were short of some particular examples. We know that the calculus for functions on general time scales is complex and difficult to implement. In order to overcome obstacles, in some cases we can combine the different methods of Lyapunov to investigate the stability of the solution. The content of this paper contains two parts: the first part presents the sufficient conditions following the first approximate method for the exponential stability of the solution of the linear dynamic Equation (1) on time scales. The second one gives some specific examples for applications. Besides the part two we add a theorem about the stability of the solution following the second method of Lyapunov. This theorem can be seen as a corollary of the stable criterion which was presented in [3].

\section{Main Results}

\subsection{The Stability of Linear Dynamic Equation under Perturbation on Time Scales}

Consider the dynamic equation

$$
x^{\Delta}(t)=A(t) x(t)+f(t, x), t \in T_{t_{0}}^{+},
$$

where $x(.) \in R^{n}, A(.) \in C_{r d}\left(T_{t_{0}}^{+}, M_{n}(R)\right), \quad f \in C_{r d}\left(T_{t_{0}}^{+} \times R^{n}, R^{n}\right)$ with $f(t, 0)=0$ on $T_{t_{0}}^{+}$.

In proportion to the system (4), we consider

$$
x^{\Delta}(t)=A(t) x(t), t \in T_{t_{0}}^{+},
$$

where $x(.) \in R^{n}, A(.) \in C_{r d}\left(T_{t_{0}}^{+}, M_{n}(R)\right)$.

We assume that $A(t)$ is regressive $\left(\operatorname{det}[I+\mu(t) A(t)] \neq 0, \forall t \in T_{t_{0}}^{+}\right)$. We denote $\phi(t)=\phi_{A}\left(t, t_{0}\right)$ is exponential matrix of (5) with $x\left(t_{0}\right)=x_{0}$.

We easily verify that $\phi_{A}^{-1}\left(t, t_{0}\right)=\phi_{A}\left(t_{0}, t\right)$ and $\phi_{A}\left(\sigma(t), t_{0}\right)=[I+\mu(t) A(t)] \phi_{A}\left(t, t_{0}\right)$.

Theorem 4. We assume that the trivial solution of (5) is exponentially stable, there exists $K>0,-\lambda \in S(T)$ to satisfy

$$
\left\|\phi_{A}\left(t, t_{0}\right)\right\| \leq K e_{-\lambda}\left(t, t_{0}\right), \forall t \in T_{t_{0}}^{+},
$$

then the trivial solution of (4) is exponentially stable if one of these conditions is satisfied

i)

$$
\lim _{\|x(t)\| \rightarrow 0} \frac{\|f(t, x(t))\|}{\|x(t)\|}=0, \forall t \in T_{t_{0}}^{+} .
$$

ii) There exists a function $g \in C_{r d}\left(T_{t_{0}}^{+}, R^{+}\right)$to satisfy

$$
\|f(t, x)\| \leq g(t)\|x\|,
$$

where

$$
\exp \left\{\int_{t_{0}}^{\infty} \lim _{s \rightarrow \mu(t)} \frac{\ln |1+g(t) s|}{s} \Delta t\right\}<+\infty .
$$

Proof. We assume that $x(t)$ is the solution of (4) with $x\left(t_{0}\right)=x_{0}$,

$$
x(t)=\phi_{A}\left(t, t_{0}\right) x_{0}+\int_{t_{0}}^{t} \phi_{A}(t, \sigma(s)) f(s, x(s)) \Delta s .
$$


By taking the norms of two sides, combinating the condition of the theorem, we obtain

$$
\begin{gathered}
\|x(t)\| \leq K e_{-\lambda}\left(t, t_{0}\right)\left\|x_{0}\right\|+\int_{t_{0}}^{t} K e_{-\lambda}(t, \sigma(s))\|f(s, x(s))\| \Delta s, \\
\Leftrightarrow e_{\Theta-\lambda}\left(t, t_{0}\right)\|x(t)\| \leq K\left\|x_{0}\right\|+\int_{t_{0}}^{t} \frac{K}{e_{-\lambda}\left(t, t_{0}\right) e_{-\lambda}(\sigma(s), t)}\|f(s, x(s))\| \Delta s, \\
\Leftrightarrow e_{\Theta-\lambda}\left(t, t_{0}\right)\|x(t)\| \leq K\left\|x_{0}\right\|+\int_{t_{0}}^{t} \frac{K}{e_{-\lambda}\left(\sigma(s), t_{0}\right)}\|f(s, x(s))\| \Delta s, \\
\Leftrightarrow e_{\Theta-\lambda}\left(t, t_{0}\right)\|x(t)\| \leq K\left\|x_{0}\right\|+\int_{t_{0}}^{t} \frac{K}{1-\mu(s) \lambda \mid e_{-\lambda}\left(s, t_{0}\right)}\|f(s, x(s))\| \Delta s .
\end{gathered}
$$

Following the assumption i), for all $\varepsilon>0$, there exists $\delta>0$ satisfies $\|f(t, x(t))\| \leq \varepsilon\|x(t)\|$ where $t \in T_{t_{0}}^{+}$, $\|x\|<\delta$. We obtain

$$
e_{\Theta-\lambda}\left(t, t_{0}\right)\|x(t)\| \leq K\left\|x_{0}\right\|+\int_{t_{0}}^{t} \frac{K \varepsilon}{|1-\mu(s) \lambda|} e_{\Theta-\lambda}\left(s, t_{0}\right)\|x(s)\| \Delta s .
$$

Let $\gamma$ is a positive satisfies $\frac{K}{|1-\mu(s) \lambda|} \leq \gamma$, put $y(t)=e_{\Theta-\lambda}\left(t, t_{0}\right)\|x(t)\|$, then

$$
y(t) \leq K\left\|x_{0}\right\|+\varepsilon \gamma \int_{t_{0}}^{t} y(s) \Delta s .
$$

By using the Gronwall inequality (see [7]), we obtain

Equivalent

$$
y(t) \leq K\left\|x_{0}\right\| e_{\varepsilon \gamma}\left(t, t_{0}\right) \leq K\left\|x_{0}\right\| \mathrm{e}^{\int_{t_{0}}^{t} \gamma \gamma \Delta s}=K\left\|x_{0}\right\| \mathrm{e}^{\varepsilon \gamma\left(t-t_{0}\right)} .
$$

$$
\begin{aligned}
& e_{\Theta-\lambda}\left(t, t_{0}\right)\|x(t)\| \leq K\left\|x_{0}\right\| \mathrm{e}^{\varepsilon \gamma\left(t-t_{0}\right)}, \\
& \Leftrightarrow\|x(t)\| \leq K\left\|x_{0}\right\| e_{-\lambda}\left(t, t_{0}\right) \mathrm{e}^{\varepsilon \gamma\left(t-t_{0}\right)} .
\end{aligned}
$$

By the assumption $-\lambda \in S(T)$, put $q:=\limsup _{\tau \rightarrow \infty} \frac{1}{\tau-t_{0}} \int_{t_{0}}^{\tau} \lim _{s \rightarrow \mu(t)} \frac{\ln |1-\lambda s|}{s} \Delta t<0$.

We obtain

$$
e_{-\lambda}\left(t, t_{0}\right) \leq \mathrm{e}^{q\left(t-t_{0}\right)}
$$

Therefore

$$
\|x(t)\| \leq K\left\|x_{0}\right\| \mathrm{e}^{q\left(t-t_{0}\right)} \mathrm{e}^{\varepsilon \gamma\left(t-t_{0}\right)}=K\left\|x_{0}\right\| \mathrm{e}^{(q+\varepsilon \gamma)\left(t-t_{0}\right)} .
$$

With $q<0$, we can choose $\varepsilon>0$, which is sufficiently small and $q+\varepsilon \gamma<0$. So that the trivial solution of (4) is exponentially stable on $T_{t_{0}}^{+}$.

For ii), by argument similarly as in i), the proof is completed.

\subsection{The Stability of Scalar Dynamic Equation on Time Scales}

For convenience, the first we consider the scalar dynamic equation

$$
x^{\Delta}(t)=p(t) x(t)+f(t, x), t \in T_{t_{0}}^{+},
$$

where $p \in C_{r d}\left(T_{t_{0}}^{+}, R\right), f \in C_{r d}\left(T_{t_{0}}^{+} \times R, R\right), f(t, 0)=0$.

Theorem 5. We assume that $p \in C_{r d}\left(T_{t_{0}}^{+}, R\right)$ satisfies the condition

$$
\limsup _{\tau \rightarrow \infty} \frac{1}{\tau-t_{0}} \int_{t_{0}}^{\tau} \lim _{s \rightarrow \mu(t)} \frac{\ln |1+p(t) s|}{s} \Delta t<0 .
$$


Then the trivial solution of (6) is exponentially stable if one of these conditions is satisfied

i)

$$
\lim _{x \rightarrow 0} \frac{|f(t, x(t))|}{|x(t)|}=0, \forall t \in T_{t_{0}}^{+} .
$$

ii) There exists a function $g \in C_{r d}\left(T_{t_{0}}^{+}, R^{+}\right)$to satisfy

$$
|f(t, x)| \leq g(t)|x|
$$

where

$$
\exp \left\{\int_{t_{0}}^{\infty} \lim _{s \rightarrow \mu(t)} \frac{\ln |1+g(t) s|}{s} \Delta t\right\}<+\infty .
$$

Proof. Let $x(t)=x\left(t, t_{0}, x_{0}\right)$ is the solution of (6) with $x\left(t_{0}\right)=x_{0}$, we have

$$
x(t)=e_{p}\left(t, t_{0}\right) x_{0}+\int_{t_{0}}^{t} e_{p}(t, \sigma(s)) f(s, x(s)) \Delta s .
$$

By taking two sides

$$
|x(t)| \leq e_{p}\left(t, t_{0}\right)\left|x_{0}\right|+\int_{t_{0}}^{t} e_{p}(t, \sigma(s))|f(s, x(s))| \Delta s .
$$

By argument similarly as the proof in theorem 4, we obtain results.

In the next part, for convenience to investigate the stability in specific examples, we represent a theorem about the sufficient condition for the exponential stability of the trivial solution of system (2). This result can be seen as a corollary of the stable criterion B. Kaymakcalan (see [3]).

We assume $V: T_{t_{0}}^{+} \times R^{n} \rightarrow R^{+}$is Delta differential of $t$, continuous differential of $x$ and $x=x\left(t, t_{0}, x_{0}\right)$ is the solution of (2) with $x\left(t_{0}\right)=x_{0}$. Then derivative of $V(t, x)$ following the trajectory of $x(t)$ is defined by $V^{\Delta}(t, x)=V^{\Delta}(t, x(t))$ and

$$
\begin{aligned}
V^{\Delta}(t, x) & =V_{t}^{\Delta}(t, x(\sigma(t)))+\int_{0}^{1} V_{x}^{\prime}\left(t, x(t)+h \mu(t) x^{\Delta}(t)\right) \mathrm{d} h x^{\Delta}(t) \\
& =V_{t}^{\Delta}(t, x(\sigma(t)))+\int_{0}^{1} V_{x}^{\prime}(t, x(t)+h \mu(t) F(t, x)) \operatorname{d} h F(t, x) .
\end{aligned}
$$

Function $V(t, x)$ with above properties is a Lyapunov function.

Theorem 6. We assume that there exists function $V: T_{t_{0}}^{+} \times R^{n} \rightarrow R^{+}$is a Lyapunov function which satisfies the following conditions

$$
\begin{gathered}
\lambda_{1}\|x\|^{\alpha} \leq V(t, x), \\
V^{\Delta}(t, x) \leq g(t, V(t, x)),
\end{gathered}
$$

where $\lambda_{1}>0$ and $\alpha \geq 1$ are positive real numbers, $g \in C_{r d}\left(T_{t_{0}}^{+} \times R^{+}, R\right)$.

If the trivial solution of

$$
u^{\Delta}=g(t, u) \text {, }
$$

is exponentially stable then the trivial solution of (2) is also exponentially stable.

Proof. By the assumption the trivial solution of (7) is exponentially stable, then the maximal solution $r(t)$ of (7) with $u\left(t_{0}\right)=u_{0} \geq 0$ satisfies

$$
|r(t)| \leq N \mathrm{e}^{-\gamma\left(t-t_{0}\right)}, t \in T_{t_{0}}^{+},
$$

where $N>0$ and $-\gamma \in S(T)$. By theorem 2.1 (see [3]) we obtain

$$
V(t, x) \leq|r(t)| \leq N \mathrm{e}^{-\gamma\left(t-t_{0}\right)}, t \in T_{t_{0}}^{+} .
$$

Using the assumption, we have 


$$
\lambda_{1}\|x\|^{\alpha} \leq V(t, x) \leq N \mathrm{e}^{-\gamma\left(t-t_{0}\right)}, t \in T_{t_{0}}^{+} .
$$

Therefore

$$
\|x\| \leq\left(\frac{N}{\lambda_{1}}\right)^{\frac{1}{\alpha}} \mathrm{e}^{-\frac{\gamma}{\alpha}\left(t-t_{0}\right)}, t \in T_{t_{0}}^{+} .
$$

By the assumption $\alpha \geq 1$ implies the trivial solution of (2) is exponentially stable.

\section{Applications}

In this part, we represent some examples of applications.

Example 1. Assume that $\alpha, \beta$ are positive constants. These functions $f_{i} \in C_{r d}\left(T_{t_{0}}^{+} \times R^{2}, R\right) ; i=1,2$ satisfy one of the conditions i) or ii) of theorem 4 . Consider system

$$
\left\{\begin{array}{l}
x_{1}^{\Delta}(t)=-\alpha x_{1}-\beta x_{2}+f_{1}\left(t, x_{1}, x_{2}\right) \\
x_{2}^{\Delta}(t)=\beta x_{1}-\alpha x_{2}+f_{2}\left(t, x_{1}, x_{2}\right)
\end{array} .\right.
$$

We assume that $f_{i}(t, 0,0)=0, \forall t \in T_{t_{0}}^{+}$in order that system (8) has the trivial solution. We consider

$$
\left\{\begin{array}{l}
x_{1}^{\Delta}(t)=-\alpha x_{1}-\beta x_{2} \\
x_{2}^{\Delta}(t)=\beta x_{1}-\alpha x_{2}
\end{array} .\right.
$$

In order to investigate the stability of (9), we choose Lyapunov function $V\left(x_{1}, x_{2}\right)=x_{1}^{2}+x_{2}^{2}$.

Taking Delta derivative, we obtain

$$
V^{\Delta}=2 x_{1}^{\Delta}(t) x_{1}(t)+2 x_{2}^{\Delta}(t) x_{2}(t)+\mu(t)\left[\left(x_{1}^{\Delta}(t)\right)^{2}+\left(x_{2}^{\Delta}(t)\right)^{2}\right] .
$$

Therefore the derivative of right-hand side of (9) is

$$
V^{\Delta}\left(t, x_{1}, x_{2}\right)=\left[-2 \alpha+\mu(t)\left(\alpha^{2}+\beta^{2}\right)\right]\left(x_{1}^{2}+x_{2}^{2}\right),
$$

which implies if $\mu(t)<\frac{2 \alpha}{\alpha^{2}+\beta^{2}}$ then the trivial solution of scalar dynamic equation

$$
u^{\Delta}=-2 \alpha u+\mu(t)\left(\alpha^{2}+\beta^{2}\right) u
$$

is exponentially stable.

By using the results of theorem 6, the trivial solution of (9) is exponentially stable.

Therefore following theorem 4 , the trivial solution of (8) is exponentially stable.

Example 2. Consider system

$$
\left\{\begin{array}{l}
x^{\Delta}(t)=-x-y+z+y^{2}+x z \\
y^{\Delta}(t)=x-y-z-x y-z^{2} \\
z^{\Delta}(t)=-x+y-z-x^{2}+y z
\end{array} .\right.
$$

In proportion to system (10), we investigate the stability of the trivial solution of system

$$
\left\{\begin{array}{l}
x^{\Delta}(t)=-x-y+z \\
y^{\Delta}(t)=x-y-z \\
z^{\Delta}(t)=-x+y-z
\end{array}\right.
$$

We choose Lyapunov function $V(x, y, z)=x^{2}+y^{2}+z^{2}$, we obtain 


$$
\begin{aligned}
V^{\Delta} & =2 x^{\Delta}(t) x(t)+2 y^{\Delta}(t) y(t)+2 z^{\Delta}(t) z(t)+\mu(t)\left[\left(x^{\Delta}(t)\right)^{2}+\left(y^{\Delta}(t)\right)^{2}+\left(z^{\Delta}(t)\right)^{2}\right] \\
& =-2\left(x^{2}+y^{2}+z^{2}\right)+\mu(t)\left[3\left(x^{2}+y^{2}+z^{2}\right)-2(x y+y z+z x)\right] \\
& =-2\left(x^{2}+y^{2}+z^{2}\right)+\mu(t)\left[4\left(x^{2}+y^{2}+z^{2}\right)-(x+y+z)^{2}\right] .
\end{aligned}
$$

Therefore

$$
V^{\Delta}(t, x, y, z) \leq(-2+4 \mu(t))\left(x^{2}+y^{2}+z^{2}\right)
$$

which implies if $\mu(t)<\frac{1}{2}$ then the trivial solution of scalar dynamic equation

$$
u^{\Delta}=(-2+4 \mu(t)) u,
$$

is exponentially stable.

By using the results of theorem 6, the trivial solution of (11) is exponentially stable.

Consider function

$$
\begin{gathered}
f(x, y, z)=\left(y^{2}+x z,-x y-z^{2},-x^{2}+y z\right), \\
\|f(x, y, z)\|^{2}=\left(y^{2}+x z\right)^{2}+\left(x y+z^{2}\right)^{2}+\left(-x^{2}+y z\right)^{2} .
\end{gathered}
$$

By taking the right-hand side, we obtain

$$
\left(y^{2}+x z\right)^{2}=(y y+x z)^{2} \leq\left(y^{2}+x^{2}\right)\left(y^{2}+z^{2}\right) \leq\left(x^{2}+y^{2}+z^{2}\right)\left(x^{2}+y^{2}+z^{2}\right)=\left(x^{2}+y^{2}+z^{2}\right)^{2} .
$$

By argument similarly as the above inequality

$$
\begin{aligned}
& \left(x y+z^{2}\right)^{2} \leq\left(x^{2}+y^{2}+z^{2}\right)^{2}, \\
& \left(-x^{2}+y z\right)^{2} \leq\left(x^{2}+y^{2}+z^{2}\right)^{2},
\end{aligned}
$$

which implies

$$
\begin{gathered}
\|f(x, y, z)\|^{2} \leq 3\left(x^{2}+y^{2}+z^{2}\right)^{2}, \\
\Leftrightarrow\|f(x, y, z)\| \leq \sqrt{3}\left(x^{2}+y^{2}+z^{2}\right), \\
\Leftrightarrow \frac{\|f(x, y, z)\|}{\sqrt{x^{2}+y^{2}+z^{2}}} \leq \sqrt{3\left(x^{2}+y^{2}+z^{2}\right)} .
\end{gathered}
$$

Therefore

$$
\lim _{x, y, z \rightarrow 0} \frac{\|f(x, y, z)\|}{\sqrt{x^{2}+y^{2}+z^{2}}}=0,
$$

by using theorem 4 , which implies the trivial solution of system (10) is exponentially stable.

\section{References}

[1] Hilger, S. (1990) Analysis on Measure Chains_A Unified Approach to Continuous and Discrete Calculus. Results in Mathematics, 18, 19-56. http://dx.doi.org/10.1007/BF03323153

[2] Bohner, M. and Peterson, A. (2001) Dynamic Equation on Time Scales: An Introduction with Applications. Birkhauser, Boston. http://dx.doi.org/10.1007/978-1-4612-0201-1

[3] Kaymakacalan, B., Lakshmikantham, V. and Sivasundaram, S. (1996) Dynamic Systems on Measure Chains. Kluwer, Dordrecht. 
[4] Potzsche, C., Siegmund, S. and Wirth, F. (2003) A Spectral Characterization of Exponential Stability for Linear TimeInvariant Systems on Time Scales. Discrete and Continuous Dynamical Systems, 9, 1223-1241. http://dx.doi.org/10.3934/dcds.2003.9.1223

[5] Agarwal, R., Bohner, M., O’Regan, D. and Peterson, A. (2002) Dynamic Equation on Time Scales: A Survey. Journal of Computational and Applied Mathematics, 141, 1-26. http://dx.doi.org/10.1016/S0377-0427(01)00432-0

[6] Aulback, B. and Hilger, S. (1971) Linear Dynamic Processes with Inhomogeneous Time Scale. In: Nonlinear Dynamics and Quantum Dynamical Systems, 3rd Edition, Akademie-Verlag, Berlin, Mathematical Research Bd. 59.

[7] Akin-Bohner, E., Bohner, M. and Akin, F. (2005) Pachpatte Inequalities on Time Scales. Journal of Inequalities in Pure and Applied Mathematics, 6, 23. 
Scientific Research Publishing (SCIRP) is one of the largest Open Access journal publishers. It is currently publishing more than 200 open access, online, peer-reviewed journals covering a wide range of academic disciplines. SCIRP serves the worldwide academic communities and contributes to the progress and application of science with its publication.

Other selected journals from SCIRP are listed as below. Submit your manuscript to us via either submit@scirp.org or Online Submission Portal.
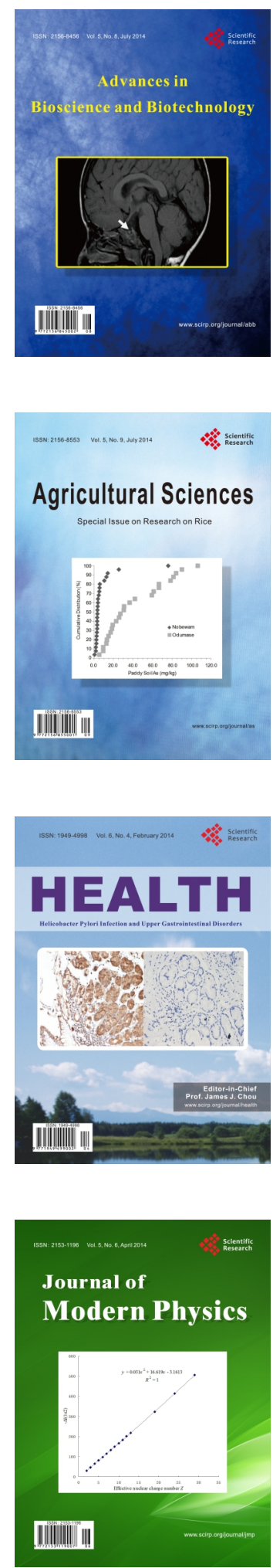
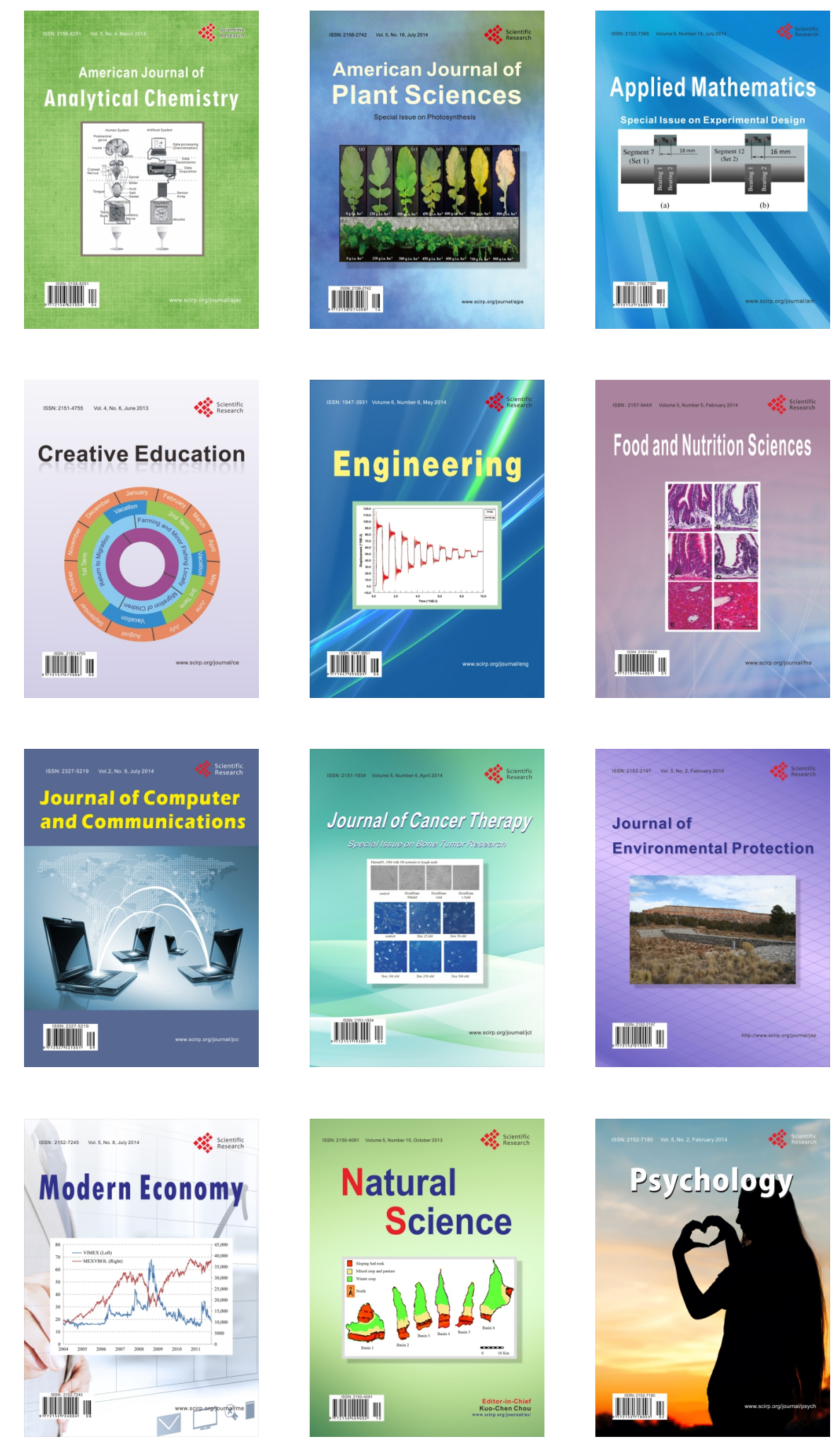